\title{
UNITARY CHERN-SIMONS MATRIX MODEL AND THE VILLAIN LATTICE ACTION
}

\author{
MAURICIO ROMO AND MIGUEL TIERZ
}

\begin{abstract}
We use the Villain approximation to show that the Gross-Witten model, in the weak- and strong-coupling limits, is related to the unitary matrix model that describes $U(N)$ Chern-Simons theory on $S^{3}$. The weak-coupling limit corresponds to the $q \rightarrow 1$ limit of the Chern-Simons theory while the strong-coupling regime is related to the $q \rightarrow 0$ limit. In the latter case, there is a logarithmic relationship between the respective coupling constants. We also show how the Chern-Simons matrix model arises by considering two-dimensional YangMills theory with the Villain action. This leads to a $U(1)^{N}$ theory which is the Abelianization of $2 \mathrm{~d}$ Yang-Mills theory with the heat-kernel lattice action. In addition, we show that the character expansion of the Villain lattice action gives the q deformation of the heat kernel as it appears in $q$-deformed 2d Yang-Mills theory. We also study the relationship between the unitary and Hermitian Chern-Simons matrix models and the rotation of the integration contour in the corresponding integrals.
\end{abstract}

\section{INTRODUCTION}

In the 1970s, Wilson introduced and studied lattice versions of Yang-Mills (YM) theory, in which the dynamical variables are elements of the gauge group, defined on the links that connect the adjacent lattice points [1]. The choice of the lattice action in Yang-Mills theory was an important aspect of the problems considered in the early development of lattice gauge theory [1]-9. In particular, in the late 1970s and early 1980s, a number of papers studied this possibility, leading to the consideration of several alternatives to the Wilson action [1, like the heat-kernel action [2, 3, 4, or the Manton action [5. One of the motivations was the need for a proper understanding of the transition from strong to weak coupling in lattice gauge theories.

A well-known result in the study of non-Abelian two-dimensional Yang-Mills theory with the Wilson action is the third-order phase transition, found by Gross and Witten [10] and Wadia [11, in a one-plaquette model, described by a unitary matrix model. This result has turned out to be of relevance in many current problems in theoretical physics, like the study of Hagedorn and deconfining transitions in weakly coupled Yang-Mills theory [12]. The Gross-Witten model has been also recently discussed in the study of type 0B and 0A fermionic string theories [13] and in relation with other solvable models, like the Kontsevich model [14].

We will first show that the unitary matrix model of $U(N)$ Chern-Simons theory on $S^{3}[15$, [16. 17] is intimately related to the Gross-Witten model when one considers it together with the Villain approximation of the XY model [18, 19]. We introduce the Villain approximation, together with the two relevant matrix models, in the next section. We will show that both the weak-coupling and strong-coupling regimes of the Gross-Witten model can be described, using the Villain approximation, by analytically continued $U(N)$ Chern-Simons on $S^{3}$. Recall that the Chern-Simons action is given by [20]

$$
S_{\mathrm{CS}}(A)=\frac{k}{4 \pi} \int_{M} \operatorname{Tr}\left(A \wedge d A+\frac{2}{3} A \wedge A \wedge A\right),
$$

where $A$ is the connection, a 1-form valued on the corresponding Lie algebra, and $k \in \mathbb{Z}$ is the level. The $q$-parameter is defined in terms of the level $k$ by $q=\exp (2 \pi i /(k+N))$. 
We will see that the weak-coupling limit corresponds to the $q \rightarrow 1$ limit of the Chern-Simons theory, whereas the strong-coupling limit of the Gross-Witten model corresponds to the opposite limit, $q \rightarrow 0$. In the matrix model formulation, the $q$ parameter is treated as real and written in terms of a coupling constant $g_{s}$ as $q=\mathrm{e}^{-g_{s}}$ [21]. The above characterization of Chern-Simons theory as being analytically continued precisely refers to this treatment of $q$ as a real parameter.

Indeed, actual computations with the matrix model are carried out with $q$ real, using for example the associated $q$-orthogonal polynomials [21], and the identification $g_{s}=2 \pi i /(k+N)$ at the end, allows us to make contact with the well-known expressions for the Chern-Simons observables [20]. See for example [21], where the simple case of the $U(N)$ Chern-Simons partition function on $S^{3}$ is computed with the Stieltjes-Wigert polynomials with a $q$ parameter, $q=\mathrm{e}^{-g_{s}}$.

The correspondence between the Gross-Witten model and the Chern-Simons matrix model is of a rather different nature in the two opposite limits $q \rightarrow 0$ and $q \rightarrow 1$. In the weak-coupling limit $g_{\mathrm{YM}}^{2} \rightarrow 0$, as shown already in [22], the coupling constants are related by $g_{\mathrm{YM}}^{2}=2 g_{s}$, whereas in the strong-coupling limit we will have that $g_{\mathrm{s}}=2 \ln \left(g_{\mathrm{YM}}^{2}\right)$ or, equivalently, $q=1 / g_{\mathrm{YM}}^{4}$, as we shall see in Sec. 2 in detail.

We will end Sec. 2 by exploring some consequences of these relationships between the models. In particular, in Sec. 2.1 we show that the Gross-Witten model at weak coupling is a Gaussian matrix model whose free energy has an expansion that can be interpreted in terms of closed strings.

The relationship between the Gross-Witten model and the Chern-Simons matrix model, based on the application of the Villain approximation to the Wilson action, indicates that the direct consideration of the Abelian Villain action in lattice two-dimensional Yang-Mills theory, should describe Chern-Simons theory. Two-dimensional Yang-Mills theory was also studied with the Manton action [23, 24] and the heat-kernel action [4]. We shall see that, indeed, the straightforward generalization of the Abelian $U(1)$ lattice action, which is just a theta function [18, 8, 7]

$$
\exp \left(-S_{\mathrm{V}}(\phi)\right)=\sum_{l=-\infty}^{\infty} \mathrm{e}^{-\frac{1}{g^{2}}(\phi+2 \pi l)^{2}},
$$

to the non-Abelian case, in the setting of $U(N)$ two-dimensional Yang-Mills theory, directly gives $U(N)$ Chern-Simons theory on $S^{3}$. This straightforward extension of the Villain lattice action to the non-Abelian case was explored by Onofri, shortly after the study of the heat-kernel case [4], in a less well-known work [9]. The description of pure Chern-Simons theory by such a model has not hitherto been realized.

It is well known that the Villain model arises in the Kogut-Susskind Hamiltonian lattice gauge theory [25] in the Abelian case, which leads to a direct correspondence with the planar Heisenberg (or XY) model [6, 2]. The non-Abelian case leads to the heat kernel [2, 4], and we shall see that the Chern-Simons matrix model follows from Abelianization of the heat-kernel propagator in the context of two-dimensional Yang-Mills theory. This is the content of Sec. 3 and, in particular, we show in Sec. 3.1 that this Abelian projection is equivalent to a $q$ deformation of 2d Yang-Mills, in consistency with the known relationship between Chern-Simons theory and a $q$ deformation of 2d Yang-Mills theory [26]. Recall that Chern-Simons theory is known to be explained in terms of an Abelian two-dimensional Yang-Mills theory, as was shown at the level of the path integral, first in the case of manifolds of the type $S^{1} \times \Sigma_{h}$, where $\Sigma_{h}$ denotes a Riemann surface of genus $h$ [27] and, more recently, for Seifert fibrations over $\Sigma_{h}$ [28], which contains the $S^{3}$ case, the one studied in this paper at the level of the matrix model. 
To conclude, we study in the Appendix the precise relationship between the unitary and the Hermitian versions of the Chern-Simons matrix model focusing also in the rotation of the contours of integration.

\section{Gross-Witten model and the Villain approximation}

The approximation devised by Villain in 1975 in the study of the two-dimensional XY model [18] is based on the simple observation that the term $\exp (\beta \cos \theta)$ that appears in the $2 \mathrm{~d} X Y$ model can be well approximated for large $\beta$ by a periodic Gaussian with minima in the same locations and with the same curvature. That is

$$
\exp (\beta \cos \theta) \sim \mathrm{e}^{\beta} \sum_{n=-\infty}^{\infty} \mathrm{e}^{-\frac{1}{2} \beta(\theta-2 \pi n)^{2}} \text { for } \beta \rightarrow \infty .
$$

But the l.h.s. term is of course also the weight function of the matrix model description of the one-plaquette model of Yang-Mills theory based on the Wilson action (namely, the Gross-Witten model [10]). The r.h.s is a theta function and then the Villain approximation applied to the Gross-Witten model leads to the relationship with a unitary matrix model with a theta function as weight function.

Precisely, the unitary matrix model that describes $U(N)$ Chern-Simons theory on $S^{3}$ [15, 16, 17. is given by 1

$$
Z_{\mathrm{CS}}^{U(N)}\left(S^{3}\right)=\int_{0}^{2 \pi} \prod_{j=1}^{N} \frac{\mathrm{d} \theta_{j}}{2 \pi} \Theta\left(\mathrm{e}^{\mathrm{i} \theta_{j}} \mid q\right) \prod_{k<l}\left|\mathrm{e}^{\mathrm{i} \theta_{k}}-\mathrm{e}^{\mathrm{i} \theta_{l}}\right|^{2},
$$

where the weight function of the matrix model is a Jacobi third theta function

$$
\omega(\theta)=\Theta\left(\mathrm{e}^{\mathrm{i} \theta_{j}} \mid q\right)=\sum_{n=-\infty}^{\infty} \mathrm{q}^{n^{2} / 2} \mathrm{e}^{i n \theta}
$$

We show now how this model follows from the Gross-Witten model [10, 11, by using the Villain approximation [19]. Aspects of the relationship between the two models, especially in the weakcoupling regime, have already been studied in [22], using orthogonal polynomials. The results of the seminal works [18, 19] also allow us to extend the relationship between the Gross-Witten and the Chern-Simons models to the strong-coupling regime.

Recall that the Gross-Witten model is a unitary one-matrix model which arises as the oneplaquette reduction of the combinatorial quantization of Yang-Mills theory. In two dimensions the reduction is exact and described by the partition function [10]

$$
\begin{aligned}
Z_{N}^{\mathrm{GW}}(\beta) & :=\int_{U(N)} \mathrm{d} U \exp \left(\frac{\beta}{2} \operatorname{Tr}\left(U+U^{\dagger}\right)\right) \\
& =\int_{0}^{2 \pi} \prod_{i=1}^{N} \mathrm{~d} \theta_{i} \mathrm{e}^{\beta \cos \theta_{i}} \prod_{i<j} \sin ^{2}\left(\frac{\theta_{i}-\theta_{j}}{2}\right),
\end{aligned}
$$

where $\mathrm{d} U$ denotes the bi-invariant Haar measure for integration over the unitary group $U(N)$. The $\beta$ parameter is usually written in terms of the gauge coupling constant as $\beta=2 / g_{\mathrm{YM}}^{2}$ [10] and hence, the strong coupling limit is given by $\beta \rightarrow 0$ and the weak coupling limit by $\beta \rightarrow \infty$.

\footnotetext{
${ }^{1}$ The unitary matrix model $(2.2)$ also describes Chern-Simons theory if the weight function is $\Theta^{-1}\left(-\mathrm{e}^{\mathrm{i} \theta_{j}} \mid q\right)$ [16. This possibility has also been noticed in [17]. The nonuniqueness description of the Chern-Simons matrix models is described in 21. See the Appendix for its precise relationship with the Hermitian matrix model
} 
The planar or XY model is characterized by a coupling between nearest-neighbor spins which has the same analytical form as the potential in the Gross-Witten model [18, 19]

$$
V_{\beta}=-\beta\left[1-\cos \left(\theta-\theta^{\prime}\right)\right] .
$$

The coefficients of the Fourier expansion of this potential are given by

$$
\mathrm{e}^{\widetilde{V}(s)}=I_{s}(\beta),
$$

where $I_{n}(z)$ is the modified Bessel function of order $n$. The partition function of the GrossWitten model can also be written as the determinant of a Toeplitz matrix with (2.6) as entries of the matrix [29]

$$
Z_{N}(\beta)=\operatorname{det}_{1 \leq i, j \leq N}\left[I_{i-j}(2 \beta)\right] .
$$

On the other hand, for $U(N)$ Chern-Simons theory on $S^{3}$ the corresponding Toeplitz determinant is $[16]^{2}$

$$
Z_{\mathrm{CS}}^{U(N)}\left(S^{3}\right)=\operatorname{det}_{1 \leq i, j \leq N}\left[a_{i-j}(q)\right], \quad \text { with } \quad a_{j}=q^{j^{2} / 2} .
$$

In the weak-coupling limit $\beta \rightarrow \infty$, the Fourier coefficient is

$$
\lim _{\beta \rightarrow \infty} \mathrm{e}^{\widetilde{V}(s)}=\mathrm{e}^{-s^{2} / 2 \beta}=\mathrm{e}^{-s^{2} g_{\mathrm{YM}}^{2} / 4} .
$$

In this limit, the Toeplitz determinant (2.7) has the Gaussian coefficients (2.9) as entries, and hence it coincides with (2.8). Of course, $g_{\mathrm{YM}}^{2} \rightarrow 0$ in (2.9) and this implies $g_{s} \rightarrow 0$ on the Chern-Simons theory side as well.

The prescription in [18, namely (2.1), is valid for both the opposite $\beta \rightarrow 0$ and $\beta \rightarrow \infty$ limits, using always periodic Gaussians, but including a renormalization scale $R_{V}(\beta)$ and a rescaled inverse temperature $\beta_{V}=f(\beta)$. This implies that a correspondence between the Gross-Witten model and the Chern-Simons matrix model holds for both the weak-coupling and the strongcoupling regimes of the model. As seen above with the Toeplitz determinant representation of the matrix model, and also in 22 using orthogonal polynomials, the weak-coupling limit follows in a straightforward way. This result, as shown in [19], also follows by considering decimation 30. It is explained in [19 that, after a few iterations of the Kadanoff-Migdal decimation procedure, any interaction function at reasonably low temperatures generates a new interaction of the Villain type

$$
\mathrm{e}^{V_{V}\left(\theta-\theta^{\prime}\right)}=\sum_{m=-\infty}^{\infty} \mathrm{e}^{-\beta_{V}\left(\theta-\theta^{\prime}-2 \pi m\right)^{2} / 2} .
$$

In addition, the critical properties of the two models could be identical by conveniently choosing $\beta_{V}=f(\beta)$ a function of $\beta$ [19]. In particular, a comparison of the interaction form for weak and strong coupling showed equivalence if

$$
\begin{array}{ll}
f(\beta)=\beta & \text { for } \beta \rightarrow \infty \\
f(\beta)=[2 \ln (2 / \beta)]^{-1} & \text { for } \beta \rightarrow 0 .
\end{array}
$$

Notice that to compare with the theta function as it appears in the matrix model (2.2), we have to take into account that

$$
\sum_{n=-\infty}^{\infty} \mathrm{e}^{-\beta(\theta+2 \pi n)^{2}}=\frac{1}{\sqrt{4 \pi \beta}} \sum_{n=-\infty}^{\infty} \mathrm{e}^{-n^{2} /(4 \beta)} \mathrm{e}^{i n \theta} .
$$

\footnotetext{
${ }^{2}$ Such a determinant was already considered in 9 . See Sec. 3.
} 
The r.h.s. of (2.11) is the series expansion of the theta function in (2.2). Thus, the decimation of the weight function of the Gross-Witten model coincides with the Villain approximation and it leads to the weight function of the unitary Chern-Simons matrix model (2.2). In the weakcoupling limit $\beta \rightarrow \infty$, we obtain that $g_{s}=1 / \beta$ and hence that $g_{s}=g_{\mathrm{YM}}^{2} / 2$. This also follows from (2.9).

The strong-coupling regime $\beta \rightarrow 0$ is specially interesting. Notice that taking $\beta=0\left(g_{Y M}^{2}=\right.$ $\infty)$ directly leads to the circular ensemble [31]

$$
Z_{N}^{\mathrm{GW}}(\beta=0)=\int_{0}^{2 \pi} \prod_{i<j} \sin ^{2}\left(\frac{\theta_{i}-\theta_{j}}{2}\right) \prod_{i=1}^{N} \mathrm{~d} \theta_{i} .
$$

In [22], the relationship between this model and Chern-Simons theory was studied. The result (2.10) leads to a refined understanding of this limit $\beta \rightarrow 0$. In particular, the relationship between the coupling constants is now logarithmic $g_{s}=2 \ln (2 / \beta)=2 \ln \left(g_{\mathrm{YM}}^{2}\right)$ and hence, the coupling constant of the Gross-Witten model is related to the $q$ parameter of Chern-Simons theory $q=1 / g_{\mathrm{YM}}^{4}$. To summarize, including the prefactors

$$
\begin{aligned}
& \mathrm{e}^{\beta \cos \theta} \approx \frac{\mathrm{e}^{\beta}}{\sqrt{2 \pi \beta}} \Theta\left(\mathrm{e}^{\mathrm{i} \theta_{j}} \mid \mathrm{e}^{-1 / \beta}\right), \text { with } g_{s}=g_{\mathrm{YM}}^{2} / 2 \text { for } g_{\mathrm{YM}} \rightarrow 0, \\
& \mathrm{e}^{\beta \cos \theta} \approx \Theta\left(\mathrm{e}^{\mathrm{i} \theta_{j}} \mid \mathrm{e}^{-1 / \beta_{\mathrm{V}}}\right) \text {, with } g_{s}=2 \log \left(g_{\mathrm{YM}}^{2}\right) \text { for } g_{\mathrm{YM}} \rightarrow \infty .
\end{aligned}
$$

It is also worth mentioning that, already in the original paper on the XY model [18], an approximation valid for both limits was also given

$$
\mathrm{e}^{\beta \cos \theta} \approx R_{V}(\beta) \sum_{m=-\infty}^{\infty} \mathrm{e}^{-\beta_{V}(\theta-2 \pi m)^{2} / 2},
$$

with

$$
\begin{aligned}
& R_{V}(\beta)=I_{0}(\beta) \sqrt{2 \pi \beta_{V}} \\
& \mathrm{e}^{-\beta_{V} / 2}=I_{1}(\beta) / I_{0}(\beta),
\end{aligned}
$$

where $I_{1}(\beta)$ and $I_{0}(\beta)$ are, as in (2.6), Bessel functions. These are the first two Fourier coefficients of the expansion of the l.h.s. of (2.13). The values for the prefactor $R_{V}(\beta)$ and the coupling constant $\beta_{V}$ are then found by imposing the first two Fourier coefficients of the two expressions in (2.13) to coincide. The limits $\beta \rightarrow 0$ and $\beta \rightarrow \infty$ of (2.14) coincide with the previous results. It was shown in [18, that this approximation is rather good, even for values close to the critical temperature. This approximation has been studied in further detail in [32], where it was found that the convergence is better for the strongly coupled regime $\beta \rightarrow 0$. In addition, the result (2.13) can be extended, with good convergence in both limits, to the case where the original function is $\omega(\theta)=\beta \cos \theta+\gamma \cos (2 \theta)$ [32]. This suggests that not only the Gross-Witten model, but more complex multicritical unitary matrix models [33], can also be expressed in terms of the unitary Chern-Simons matrix model, only with a more sophisticated expression for the coupling constant and the prefactor.

2.1. Gaussian behavior and closed string interpretation at weak coupling. The relationship between the Gross-Witten model in the weak-coupling limit and the semiclassical limit of the Chern-Simons matrix model, indicates that the Gross-Witten model in this regime should be related to closed topological strings [34]. The reason is that the semiclassical limit $(k \rightarrow \infty)$ of the $U(N)$ Chern-Simons on $S^{3}$ free energy also coincides with the nonperturbative 
part of the total free energy. The Chern-Simons free energy can be suitably expressed in terms of nonperturbative and perturbative contributions

$$
F_{\mathrm{CS}}=\log Z_{\mathrm{CS}}=F_{\mathrm{np}}+F_{\mathrm{p}}
$$

The nonperturbative contribution $F_{\mathrm{np}}$ is the logarithm of the measure factor in the path integral, which is not captured by Feynman diagrams, and it gives the exact Chern-Simons partition function in the semiclassical limit $k \rightarrow \infty$ [34, eq. (2.8)]. It has the explicit expression

$$
F_{\text {np }}=\log \left(\frac{\left(2 \pi g_{s}\right)^{N^{2} / 2}}{\operatorname{vol}(U(N))}\right) \text {. }
$$

This free energy (2.16), given by a Hermitian Gaussian matrix model, has an expansion which can be interpreted in terms of closed topological string theory on the resolved conifold geometry [34]. See [34] for equivalent string theory and gauge theory interpretations.

Due to the correspondence between the Chern-Simons and the Gross-Witten matrix models, the latter should have (2.16) as free energy in the weak-coupling limit $\beta \rightarrow \infty$. Consider the expression for the free energy of the Gross-Witten model for finite $N$ and $g_{\mathrm{YM}} \rightarrow 0$ [35]

$$
F_{\mathrm{GW}} \simeq \frac{2 N}{g_{\mathrm{YM}}^{2}}-\frac{N}{2} \ln (2 \pi)-\frac{N^{2}}{2} \ln \left(\frac{2}{g_{\mathrm{YM}}^{2}}\right)+\sum_{j=1}^{N-1} \ln j !+\mathcal{O}\left(g_{Y M}^{2}\right) .
$$

This coincides with the free energy of a Hermitian Gaussian matrix model [31]

$$
Z_{\mathrm{G}}(\beta)=\mathrm{e}^{\beta N} \int_{-\infty}^{\infty} \mathrm{e}^{-\sum_{j=1}^{N} \beta x_{j}^{2} / 2} \prod_{j<k}\left(x_{j}-x_{k}\right)^{2} .
$$

This relationship between the Gross-Witten and the Gaussian matrix model agrees with [36]. Notice that the term $\mathrm{e}^{\beta N}$ actually corresponds to the $\mathrm{e}^{\beta}$ term in the Villain approximation (2.12) and implies that one has to consider the Gross-Witten model with the potential (2.5) ${ }^{3}$, instead of the one in (2.4).

\section{The Villain lattice action and Abelian/q-Deformed 2D Yang-Mills theory}

We begin by discussing the heat-kernel action, which was introduced in lattice gauge theory, at least in part, as an alternative to the Wilson action that also provided a natural extension to the non-Abelian case [2, 3, 4] of the Villain approximation of the two-dimensional XY model [18, which had been crucially used in the study of $U(1)$ lattice gauge theories [8, 7].

The setting is quantum Yang-Mills theory with gauge group $U(N)$ on an oriented closed Riemann surface $\Sigma_{h}$ of genus $h$ and unit area form $\mathrm{d} \mu$ [37]. The action is

$$
S_{\mathrm{YM}}=-\frac{1}{4 g_{Y M}} \int_{\Sigma_{h}} \mathrm{~d} \mu \operatorname{Tr} F^{2}
$$

where $g_{s}$ plays the role of the coupling constant, $F$ is the field strength of a matrix gauge connection, and $\operatorname{Tr}$ is the trace in the fundamental representation of $U(N)$. A lattice regularization of the gauge theory relies on a triangulation of the two-dimensional manifold $\Sigma$ with group matrices situated along the edges [38]. The path integral is then approximated by the finite-dimensional unitary matrix integral

$$
\mathcal{Z}_{\mathrm{M}}=\int \prod_{\text {edges } \ell} \mathrm{d} U_{\ell} \prod_{\text {plaquettes } P} Z_{P}\left[U_{P}\right]
$$

where $\mathrm{d} U_{\ell}$ denotes Haar measure on $S U(N)$ and the holonomy $U_{P}=\prod_{\ell \in P} U_{\ell}$ is the ordered product of group matrices along the links of a given plaquette. The local factor $Z_{P}\left[U_{P}\right]$ is a

\footnotetext{
${ }^{3}$ This form of the potential corresponds exactly to the Wilson lattice action.
} 
suitable gauge invariant lattice weight that converges in the continuum limit to the Boltzmann weight for the Yang-Mills action (3.1). This expression for $\mathcal{Z}_{\mathrm{M}}$ leads to the matrix models presented in the previous section after the suitable choice of lattice action.

Let us discuss now the use of the heat kernel for the lattice weight $Z_{P}\left[U_{P}\right]$. In this case, the lattice action has many interesting features [4] and is the usual choice in two-dimensional Yang-Mills theory [37]. It leads to the well-known group theory expansion of the partition function [38, 39]

$$
\mathcal{Z}_{\mathrm{M}}=\sum_{\lambda}(\operatorname{dim} \lambda)^{2-2 h} \exp \left(-g_{Y M}^{2} C_{2}(\lambda)\right)
$$

where the sum runs through all isomorphism classes $\lambda$ of irreducible representations of the $S U(N)$ gauge group, $\operatorname{dim} \lambda$ is the dimension of the representation $\lambda$, and $C_{2}(\lambda)$ is the quadratic Casimir invariant of $\lambda$. This expression for the partition function is a particular case of the propagator [4, 37]

$$
\exp \left(-S_{\mathrm{HK}}(U)\right)=\left\langle\mathbb{I}\left|\exp \left(\frac{1}{2} g_{Y M}^{2} \Delta\right)\right| U\right\rangle \equiv K\left(U, \frac{g_{Y M}^{2}}{2}\right),
$$

which can be written as 4 ]

$$
K\left(U, \frac{g_{Y M}^{2}}{2}\right)=\sum_{\lambda} \operatorname{dim} \lambda \chi_{\lambda}(U) \exp \left(-g_{Y M}^{2} C_{2}(\lambda)\right)
$$

where the sum runs over all irreducible unitary representations of the gauge group, $\chi_{\lambda}(U)$ is the character of such a representation, $\operatorname{dim} \lambda=\chi_{\lambda}(\mathbb{I})$ its dimension and $C_{2}(\lambda)$ the Casimir of the representation. Writing (3.4) in terms of the elements of the Young tableaux that labels the representation $\lambda$ leads to a discrete matrix model representation [37].

3.0.1. $q$ deformation. In recent years, the relationship between two-dimensional Yang-Mills theory and Chern-Simons theory on Seifert manifolds has been understood in further detail [26, 40, 41] (see also [28, 42, 43]). Seifert manifolds $M(h, p)$ are nontrivial circle bundles (of monopole degree $p$ ) over two-dimensional surfaces of genus $h$. The simplest case, the trivial fibration, $M(h, 0)=\Sigma_{h} \times S^{1}$ was studied in detail in [20, 27].

Chern-Simons theory on Seifert manifolds has been the subject of much interest in the study of topological strings [4] and has a direct relationship with a $q$ deformation of the two-dimensional Yang-Mills theory discussed above, when the manifold is a sphere, $\Sigma_{0}=S^{2}$. In particular, the partition function of $q$-deformed 2d YM on a closed Riemann surface of genus $h$ is given by [26]

$$
Z_{Y M}^{q}\left(\Sigma_{h}\right)=\sum_{\lambda}\left(\operatorname{dim}_{q}(\lambda)\right)^{2-2 h} q^{\frac{p}{2} C_{2}(\lambda)}
$$

where $\operatorname{dim}_{q}(\lambda)$ is the $q$ deformation of the dimensions of $s l_{n}$ representations, i.e. the quantum dimensions $\operatorname{dim}_{q}(\lambda)$ [45], $p$ is a positive integer parameter and, as usual, $C_{2}(\lambda)$ is the Casimir of the representation $\lambda$. This is related to the partition function of Chern-Simons theory on a circle fibration (with Chern class $p$ ) over $\Sigma_{h}$, which is a Seifert space. In the case $\Sigma_{h}=S^{2}$ and $p>1$, the Seifert manifold is the lens space $S^{3} / \mathbb{Z}_{p}$. If $p=1$, then the connection is with Chern-Simons theory on $S^{3}$, the case studied here.

3.1. Abelianization and q deformation. The propagator (3.4) can be alternatively written in terms of the elements of $U(N)$, and then one obtains a unitary matrix model expression. 
When written in terms of the invariant angles of the gauge group, it is given by [4]

$$
\exp \left(-S_{\mathrm{HK}}\left(\theta_{1}, \ldots \theta_{N}\right)\right)=\mathcal{N} \sum_{\{l\}=-\infty}^{\infty} \prod_{i<j} \frac{\theta_{i}-\theta_{j}+2 \pi\left(l_{i}-l_{j}\right)}{2 \sin \left[\theta_{i}-\theta_{j}+2 \pi\left(l_{i}-l_{j}\right)\right]} \exp \left[-\frac{1}{g_{Y M}^{2}} \sum_{j=1}^{N}\left(\theta_{j}+2 \pi l_{j}\right)^{2}\right],
$$

where $\mathcal{N}$ stands for some normalization. This is a rather complex model, and therefore the heatkernel case, in contrast to the two cases studied in the previous section, is not studied with a unitary matrix model but rather with a discrete matrix model that follows from (3.3). We show now that a simplification of (3.6) leads to the Chern-Simons matrix model. The exponential part in the r.h.s. is the Abelian $U(1)$ Villain action, which is just a theta function (2.1)

$$
\exp \left(-S_{\mathrm{V}}(\theta)\right)=\sum_{l=-\infty}^{\infty} \mathrm{e}^{-\frac{1}{g_{Y M}^{2}}(\theta+2 \pi l)^{2}}
$$

and the straightforward generalization to $U(N)$ gives a propagator

$$
\begin{aligned}
\bar{K}\left(U, \frac{g_{Y M}^{2}}{2}\right) & =\sum_{\{l\}=-\infty}^{\infty} \exp \left[-\frac{1}{g_{Y M}^{2}} \sum_{j=1}^{N}\left(\theta_{j}+2 \pi l_{j}\right)^{2}\right] \\
& =\prod_{i=1}^{N} \exp \left(-S_{\mathrm{V}}\left(\theta_{i}\right)\right)=\exp \left(-S_{\text {Villain }}\left(\theta_{1}, \ldots \theta_{N}\right)\right)
\end{aligned}
$$

that defines an action which is just the direct product of the Abelian Villain action (3.7). The corresponding one-plaquette model is given by a $U(N)$ matrix integral, which is the unitary integration of the propagator. The partition function is then given by the matrix model

$$
Z_{N}=\int_{0}^{2 \pi} \prod_{i=1}^{N} \frac{\mathrm{d} \theta_{i}}{2 \pi} \sum_{n=-\infty}^{\infty} \mathrm{e}^{-\frac{1}{g_{Y M}^{2}}\left(\theta_{i}+2 \pi n\right)^{2}} \prod_{k<l}\left|\mathrm{e}^{\mathrm{i} \theta_{k}}-\mathrm{e}^{\mathrm{i} \theta_{l}}\right|^{2},
$$

which, recalling the identity (2.11), is the unitary matrix model description of $U(N)$ ChernSimons theory on $S^{3}$ (2.2) discussed so far and previously studied in [15, 16, 17]. Hence, $Z_{N}=Z_{\mathrm{CS}}^{U(N)}\left(S^{3}\right)$, by identifying $g_{s}=g_{Y M}^{2} / 2$. Thus, the Abelianization of the heat kernel (3.6), given by the straightforward extension of the $U(1)$ Abelian Villain action to the $U(1)^{N}$ case (3.8), leads to $U(N)$ Chern-Simons theory on $S^{3}$.

At the level of the matrix model, this straightforward generalization of the Villain action was already considered in an interesting paper by Onofri [9], that analyzed this problem and presented a detailed study of the matrix model (3.9). It is remarkable that the results in that paper describe pure Chern-Simons theory on $S^{3}$, with $U(N)$ and $S U(N)$, a fact that has not been pointed out so far. Some expressions in [9] are manifestly Chern-Simons observables, like the partition function of $U(N)$ Chern-Simons theory on $S^{3}$.

We have seen the Abelianization of the heat kernel, using its expression in terms of the invariant angles of the gauge group (3.6). Let us now look at the relationship between the choice of the Villain lattice action and the $q$ deformation of the heat-kernel 2d Yang-Mills theory, using instead the character expansion (3.4).

Notice that the $q$-deformed 2d Yang-Mills (3.5) is just given by the usual expression for two-dimensional Yang-Mills theory based on the heat-kernel action and defined on a compact manifold of genus $h$, but with $q$ dimensions instead of ordinary dimensions, $\operatorname{dim}(\lambda) \rightarrow \operatorname{dim}_{q}(\lambda)$. We will now show that choosing (3.8) with the Villain action (1.2) as a lattice action is actually equivalent to $q$-deformed $2 \mathrm{~d}$ Yang-Mills. Since choosing the Villain action is tantamount to an 
Abelian projection of the two-dimensional Yang-Mills theory with the heat-kernel lattice action, this implies that the Abelianization and the $q$ deformation of the latter lead to the same theory.

An expression of the type (3.5) is valid for 2d Yang-Mills on a compact manifold of genus $h$ and it can be constructed from the central heat kernel, which is also the propagator on a cylinder [37]

$$
K\left(\frac{g_{Y M}^{2}}{2}, U, U^{\prime}\right)=\sum_{\lambda} \chi_{\lambda}(U) \chi_{\lambda}\left(U^{\prime}\right) \exp \left(-g_{Y M}^{2} C_{2}(\lambda)\right)
$$

with holonomies $U$ and $U^{\prime}$ on the two disks of the cylinder and where $\chi_{\lambda}(U)$ is the character of the irreducible representation $\lambda$, as in (3.4) above. Recall that $\chi_{\lambda}(U=\mathbb{I})=\operatorname{dim}(\lambda)$. Hence, if $U=U^{\prime}=\mathbb{I}$, (3.10) is the expression for the partition function of two-dimensional Yang-Mills theory on $S^{2}$. If only $U^{\prime}=\mathbb{I}$, then it describes two-dimensional Yang-Mills on a disk

$$
K\left(\frac{g_{Y M}^{2}}{2}, U, \mathbb{I}\right)=\sum_{\lambda} \chi_{\lambda}(U) \operatorname{dim} \lambda \exp \left(-g_{Y M}^{2} C_{2}(\lambda)\right) .
$$

This is the heat kernel and it is also the amplitude of a plaquette that leads, by gluing, to (3.6) [37. Since (3.11) is also the fundamental solution of the diffusion equation, then, as is well-known, two-dimensional Yang-Mills theory based on the heat kernel can be understood as a diffusion process on the gauge group manifold.

The relationship between the Chern-Simons model constructed with the Villain action and the $q$ deformation of two-dimensional Yang-Mills theory can be readily seen by considering the character expansion of the $U(N)$ Villain action (3.8) given in [9]

$$
\frac{\mathrm{e}^{-S_{\text {Villain }}(U)}}{Z}=\sum_{m_{1} \geq m_{2} \geq \ldots \geq m_{N}=0} \chi_{\left(m_{1}, \ldots, m_{N}\right)}(U) q^{\frac{1}{2} \sum_{i=1}^{N} m_{i}^{2}} \prod_{j>i}\left(\frac{q^{m_{i}-m_{j}+j-i}-1}{q^{j-i}-1}\right),
$$

where the sum is over the integers $\left\{m_{i}\right\}$ with $i=1, \ldots, N$ and $Z$ is the partition function of the unitary matrix model, which has the explicit form

$$
Z=\left(\frac{g_{\mathrm{YM}}^{2}}{8 \pi}\right)^{\frac{N}{2}} \prod_{k=1}^{N-1}\left(1-q^{N-k}\right)^{k}, \quad q=\mathrm{e}^{-\frac{g_{\mathrm{YM}}^{2}}{2}} .
$$

This character expansion of the Villain action suggests that such an action leads to the usual propagator of two-dimensional Yang-Mills theory based on the heat kernel (3.11) but with $q$ dimensions instead of dimensions, since the last term in (3.12) gives an explicit expression for quantum dimensions. An elementary manipulation of the product shows this explicitly

$$
\prod_{j>i}\left(\frac{q^{m_{i}-m_{j}+j-i}-1}{q^{j-i}-1}\right)=\prod_{j>i} q^{\frac{m_{j}-m_{i}}{2}} \frac{\left[m_{i}-m_{j}+j-i\right]_{q}}{[j-i]_{q}}=q^{\frac{1}{2} \sum_{l=1}^{N}(N-2 l+1) m_{l}} \operatorname{dim}_{q}(\lambda),
$$

where $\lambda$ denotes the unitary irreducible representation of the gauge group, characterized by a partition whose Young tableaux has columns with $\left\{m_{i}\right\}$ boxes. The explicit expression for the quantum dimensions is given, as in [26], by

$$
\operatorname{dim}_{q}(\lambda)=\prod_{j>i} \frac{\left[m_{i}-m_{j}+j-i\right]_{q}}{[j-i]_{q}}, \text { with the } q \text {-number }[x]_{q}=\frac{q^{\frac{x}{2}}-q^{-\frac{x}{2}}}{q^{\frac{1}{2}}-q^{-\frac{1}{2}}} .
$$

Putting all together, we see that the Casimir term is manifest and appears exactly as in [26]

$$
C_{2}(\lambda)=\sum_{i=1}^{N} m_{i}^{2}+(N-2 i+1) m_{i}=\sum_{i=1}^{N} m_{i}\left(m_{i}-2 i+1\right)+N \sum_{i=1}^{N} m_{i},
$$


and hence we obtain

$$
\frac{\mathrm{e}^{-S_{\text {Villain }}(U)}}{Z}=\sum_{\lambda} \chi_{\lambda}(U) q^{\frac{1}{2} C_{2}(\lambda)} \operatorname{dim}_{q}(\lambda) .
$$

Thus, the r.h.s of (3.12) is the disk amplitude for $q$-deformed two-dimensional Yang-Mills theory with $p=1$, which, by the same procedure discussed above, and explained in detail in [37], leads to the expression for the partition function (3.5). This identity is actually a particular case of the Kostant identity, which gives a character expansion of theta functions of a lattice [46. This specific form of the identity was then rediscovered later on, in [9], in the context of the Villain lattice action, and it also appears much later in [47, where, working from the r.h.s. of (3.13) a theta function expression was found, which is, as we have seen here, the Villain lattice action. In this paper, we have also shown, by using the equivalent formulation of the lattice action in terms of the invariant angles (3.6), that it follows from taking only the Abelian part of the heat kernel.

The fact that the Abelianization and the $q$ deformation described here are equivalent is qualitatively consistent with the fact, explained for example in [48], that a $q$ deformation of a Lie group $G$ is not a group and lacks its symmetry, whereas the maximal torus $T$ of $G$ remains an ordinary symmetry group after the symmetry breaking inherent in the transformation of a Lie group into a quantum group.

To conclude, let us mention that inspection of the analogous expression for $S U(N)$ in [9] shows that $\operatorname{dim}_{q} \lambda$ can also be written as the character $\chi_{\lambda}\left(T_{q}\right)$, where $T_{q} \in S L(N, \mathbb{C})$ is given by

$\operatorname{diag}\left[q^{N-1}, q^{N-3}, \ldots, q^{3-N}, q^{1-N}\right]$. Hence, the propagator is now of the type $K\left(\frac{g^{2}}{2}, U, T_{q}\right)$ instead of (3.11), which suggests that diffusion does not take place in the whole gauge group. Indeed, the explicit form of the matrix model indicates diffusion on the maximal torus $U(1) \times \ldots \times U(1)$ of the gauge group $U(N)$. This is in agreement with a previous result that related Chern-Simons theory on $S^{3}$ with Brownian motion on the Weyl chamber of the gauge group [49].

\section{Conclusions and Outlook}

We have seen how the unitary matrix model that describes $U(N)$ Chern-Simons theory on $S^{3}$ arises from studying two-dimensional Yang-Mills theory with the Villain lattice action and we have compared it both with the Wilson and the heat-kernel lattice action cases.

Regarding the former, we have seen that the Gross-Witten model is related to the ChernSimons matrix model both in the weak-coupling and the strong-coupling regimes. As we have seen in Sec. 2.1., one of the implications is that the Gross-Witten model, which describes two-dimensional Yang-Mills theory on $\mathbb{R}^{2}$, coincides in the weak-coupling limit with the nonperturbative part of Chern-Simons theory on $S^{3}$, and consequently has the same string theory interpretation [34]. In both cases, the free energy is given by a Hermitian Gaussian matrix model. In spite of the apparent simplicity of such a matrix model, it is actually relevant in the study of subsectors of $\mathcal{N}=4$ supersymmetric gauge theory and their relationship with twodimensional Yang-Mills theory (see [50, for example). It is possible that taking into account Wilson loops in our discussion would lead to a relationship with that line of research.

Notice also that the general approximation of the Gross-Witten weight (2.13) implies that a small modification of the Gross-Witten model potential leads to the Chern-Simons matrix model. The interest of this result lies in a possible connection between the Chern-Simons matrix model and the unitary matrix models that appear in the study of phase transitions of weakly coupled gauge theories [12].

The heat-kernel has a character expansion which is the basis of the study of 2d Yang-Mills theory. However, it also can be expressed in the invariant angles of the gauge group (the unitary 
group in our case), as pointed out in [4]. The unitary matrix model that follows from this representation is not used in the heat-kernel case, due to its complexity. However, we also have seen that an Abelian projection of the heat-kernel lattice action leads to a $U(1)^{N}$ lattice action, which is the Villain lattice action. After unitary integration of the resulting propagator, the corresponding matrix model is now the $U(N)$ Chern-Simons matrix model for $S^{3}$. On the other hand, since the character expansion of the Villain lattice action gives the $q$-deformed 2d Yang-Mills propagator (Kostant-Onofri identity), we see that the Abelianization of (3.6) coincides with the $q$ deformation of (3.11), given by (3.13). In addition, it shows how the $q$ propagator directly leads to the unitary Chern-Simons matrix model, instead of the (equivalent) Chern-Simons Hermitian matrix model.

Precisely, and to conclude, in the Appendix, the relationship between the unitary and the Hermitian versions of the Chern-Simons matrix model is studied in detail, focusing also in the rotation of the contours of integration.

Acknowledgments. Thanks to Sergio Iguri and Fokko van de Bult for comments and correspondence. The work of MT has been supported by the project "Probabilistic approach to finite and infinite dimensional dynamical systems" (PTDC/MAT/104173/2008) at the Universidade de Lisboa. 


\section{ApPendix A. Unitary vs HeRmitian AND INTEGRATION CONTOURS IN MATRIX MODELS}

In this paper we have focused on the unitary matrix model that describes Chern-Simons theory on $S^{3}$. It was first considered in [15] although we have seen that it was already studied in detail in [9]. In contrast to the Hermitian matrix model, no additional work has been done using the unitary model, with the exception of its recent appearance in the study of matrix models in Donaldson-Thomas theory [17, 16. The Hermitian matrix model, in the $S^{3}$ case reads [51]

$$
Z_{\mathrm{CS}}^{U(N)}\left(S^{3}\right)=\int_{-\infty}^{\infty} \mathrm{e}^{-\sum_{j=1}^{N} x_{j}^{2} /\left(2 g_{s}\right)} \prod_{j<k}\left(2 \sinh \frac{\pi\left(x_{j}-x_{k}\right)}{2}\right)^{2} \prod_{j=1}^{N} \frac{\mathrm{d} x_{j}}{2 \pi} .
$$

Both models, the unitary (2.2) and the Hermitian (A.1), are solved with orthogonal polynomials that have the same orthogonality properties, and hence the corresponding observables of the matrix model, like the partition function for example, coincide. These polynomials are the Stieltjes-Wigert polynomials in the Hermitian case [21] and the Rogers-Szegö polynomials in the case of the unit circle [52, 22]. The connection between these two systems of orthogonal polynomials allows to explain the relationship between both matrix models, as was shown in [52. However, it is interesting to have a more immediate relationship between the models. Let us write the model (A.1) in its trigonometric version:

$$
\widetilde{Z}_{\mathrm{CS}}^{U(N)}\left(S^{3}\right)=\int_{-\infty}^{\infty} \mathrm{e}^{-\frac{1}{2 g_{s}} \sum_{j=1}^{N} u_{j}^{2}} \prod_{j<k}\left(2 \sin \frac{u_{j}-u_{k}}{2}\right)^{2} \prod_{j=1}^{N} \frac{d u_{j}}{2 \pi} .
$$

We will now show its relationship with (A.1). The first step is to relate (A.2) with the unitary model (2.2), by transforming the Vandermonde determinant

$$
\prod_{j<k}\left(2 \sin \frac{u_{j}-u_{k}}{2}\right)^{2}=\prod_{j<k}\left|\mathrm{e}^{i u_{j}}-\mathrm{e}^{i u_{k}}\right|^{2},
$$

and the weight function, making the range of integration compact and using also the identity (2.11)

$$
\begin{aligned}
\int_{-\infty}^{\infty} \prod_{j<k}\left|\mathrm{e}^{i u_{j}}-\mathrm{e}^{i u_{k}}\right|^{2} \mathrm{e}^{-\frac{1}{2 g_{s}} \sum_{j=1}^{N} u_{j}^{2}} \prod_{j=1}^{N} \frac{d u_{j}}{2 \pi} & =\frac{g_{s}^{\frac{N}{2}}}{(2 \pi)^{\frac{N}{2}}} \int_{0}^{2 \pi} \prod_{j=1}^{N} \frac{d u_{j}}{2 \pi} \sum_{n=-\infty}^{\infty} \mathrm{e}^{-\frac{n^{2} g_{s}}{2}+i n u_{j}} \prod_{j<k}\left|\mathrm{e}^{i u_{j}}-\mathrm{e}^{i u_{k}}\right|^{2} \\
& =\frac{g_{s}^{\frac{N}{2}}}{(2 \pi)^{\frac{N}{2}}} \int_{0}^{2 \pi} \prod_{j=1}^{N} \frac{d u_{j}}{2 \pi} \Theta\left(q \mid u_{j}\right) \prod_{j<k}\left|\mathrm{e}^{i u_{j}}-\mathrm{e}^{i u_{k}}\right|^{2} .
\end{aligned}
$$

Hence, we see that the trigonometric matrix model (A.2) is equivalent to the unitary ChernSimons matrix model (2.2). It is also related to (A.1) by the change of variables $x=i u$

$$
\begin{aligned}
& \int_{-\infty}^{\infty} \mathrm{e}^{-\sum_{j=1}^{N} x_{j}^{2} /\left(2 g_{s}\right)} \prod_{j<k}\left(2 \sinh \left(\frac{\pi\left(x_{j}-x_{k}\right)}{2}\right)\right)^{2} \prod_{j=1}^{N} d x_{j} \\
= & \mathrm{e}^{\frac{i \pi N(N+1)}{4}} \int_{i \infty}^{-i \infty} \mathrm{e}^{\sum_{j=1}^{N} u_{j}^{2} /\left(2 g_{s}\right)} \prod_{j<k}\left(2 \sin \left(\frac{\pi\left(u_{j}-u_{k}\right)}{2}\right)\right)^{2} \prod_{j=1}^{N} d u_{j} .
\end{aligned}
$$

However, notice that the integral in the trigonometric model (A.5) is actually over the imaginary line of the complex plane. Then, to show the equivalence of (A.1) and the unitary model (2.2), the r.h.s of (A.5) has to be equal to (A.2). What we get now is (A.2) but with the opposite sign in the weight and a different integration contour. If we were able to rotate the contour to the real axis, then the sign in the exponential of the weight can be corrected just by complex conjugation of (A.5), recalling $g_{s}$ as a purely imaginary quantity. Hence, one needs to rotate 
the contour to the real line and due to the results above mentioned, we know that this has to be the case.

This rotation of the contour is also directly related to the fact that the actual derivation of the matrix model for Seifert manifolds leads to integral expressions with contours on the complex plane [53, 51, and it is just assumed that they can be rotated into the real axis [51, leading then to expressions such as (A.1).

It is then worthwhile to examine the rotation of contours more carefully. For this, we use certain particular cases of multivariate hyperbolic hypergeometric integrals, studied in [54, that we can easily identify with the Chern-Simons matrix integrals but with complex integration contours. The integrals in [54] are written in terms of the hyperbolic Gamma function. However, due to the following property of the hyperbolic Gamma function [54]

$$
\frac{1}{\Gamma_{h}\left(z \mid \omega_{1}, \omega_{2}\right) \Gamma_{h}\left(-z \mid \omega_{1}, \omega_{2}\right)}=-4 \sin \left(\frac{\pi z}{\omega_{1}}\right) \sin \left(\frac{\pi z}{\omega_{2}}\right)
$$

it is then immediate to identify such integrals in [54 with those given by the matrix model description of Chern-Simons theory on $S^{3}$. To see that this is the case, let us first give some definitions in [54] and then we quote Proposition 5.3.19 in [54].

Definition (hook): $A$ hook $W_{\phi_{1}, \phi_{2}}$ is a contour parametrized by $W_{\phi_{1}, \phi_{2}}(s)=s \mathrm{e}^{i \phi_{1}}$ for $s \in$ $(-\infty, 0]$ and $W_{\phi_{1}, \phi_{2}}(s)=s \mathrm{e}^{i \phi_{2}}$ for $s \in[0, \infty)$. The following quantities are defined as well $\phi_{+}=\max \left(\arg \left(\omega_{1}\right), \arg \left(\omega_{2}\right)\right)$ and $\phi_{-}=\min \left(\arg \left(\omega_{1}\right), \arg \left(\omega_{2}\right)\right)$ and, in terms of $\phi_{+}$and $\phi_{-}$, the domain $\mathcal{A}_{+}$in the complex plane

$$
\mathcal{A}_{+}=\left(\phi_{+}-\pi, \frac{\phi_{+}+\phi_{-}-\pi}{2}\right) .
$$

After using (A.6), Proposition 5.3.19 reads [54]

Proposition 1: For $t \in \mathbb{Z}_{>0}$ we have

$$
\begin{aligned}
J_{N, t}\left(\omega_{1}, \omega_{2}\right) & \equiv \frac{1}{\left(\sqrt{-\omega_{1} \omega_{2}}\right)^{N} N !} \int_{C} \mathrm{e}^{\frac{t \pi i}{2 \omega_{1} \omega_{2}} \sum_{j=1}^{N} x_{j}^{2}} \prod_{j<k}(-4) \sin \left(\frac{\pi\left(x_{j}-x_{k}\right)}{\omega_{1}}\right) \sin \left(\frac{\pi\left(x_{j}-x_{k}\right)}{\omega_{2}}\right) \prod_{j} d x_{j} \\
(\mathrm{~A} .7) & =\frac{\mathrm{e}^{-\frac{i \pi N^{2}}{4}} 2^{\frac{N}{2}}}{t^{\frac{N}{2}}} \mathrm{e}^{-\frac{\pi i N^{2}(N-1)\left(\omega_{1}^{2}+\omega_{2}^{2}\right)}{6 t \omega_{1} \omega_{2}}} \prod_{j=1}^{N}\left(2 \sin \left(\frac{2 \pi j}{t}\right)\right)^{N-j}
\end{aligned}
$$

where the contour of integration $C$ is a hook $W_{\phi_{1}, \phi_{2}}$ with $\phi_{1}, \phi_{2} \in \mathcal{A}_{+}$.

This result can be extended to $t \in \mathbb{Z}_{<0}$ by using a property of invariance under complex conjugation [54]

$$
J_{N, t}\left(\omega_{1}, \omega_{2}\right)=\overline{J_{N,-t}\left(-\bar{\omega}_{1},-\bar{\omega}_{2}\right)} .
$$

To compare with the expression given by the Chern-Simons matrix model, we take $\omega_{1}=\omega_{2}=i$; therefore $\mathcal{A}_{+}=\left(-\frac{\pi}{2}, 0\right)$. We also perform the change of variables $2 \pi x_{i}=u_{i}$ and identify $t=2(k+N)$. Then the integral can be written as

$$
\int_{C} \prod_{j<k} 4 \sinh ^{2}\left(\frac{u_{j}-u_{k}}{2}\right) \mathrm{e}^{\frac{1}{2 g_{s}} \sum_{j} u_{j}^{2}} \prod_{j} \frac{d u_{j}}{2 \pi}=N ! \mathrm{e}^{-\frac{i \pi N^{2}}{4}}(k+N)^{-\frac{N}{2}} \mathrm{e}^{-\frac{g_{s} N^{2}(N-1)}{12}} \prod_{j=1}^{N}\left(2 \sin \left(\frac{\pi j}{k+N}\right)\right)^{N-j}
$$


or, equivalently [using (A.8)]

(A.10)

$$
\int_{C} \prod_{j<k} 4 \sinh ^{2}\left(\frac{u_{j}-u_{k}}{2}\right) \mathrm{e}^{-\frac{1}{2 g_{s}} \sum_{j} u_{j}^{2}} \prod_{j} \frac{d u_{j}}{2 \pi}=N ! \mathrm{e}^{\frac{i \pi N^{2}}{4}}(k+N)^{-\frac{N}{2}} \mathrm{e}^{\frac{g_{s} N^{2}(N-1)}{12}} \prod_{j=1}^{N}\left(2 \sin \left(\frac{\pi j}{k+N}\right)\right)^{N-j} .
$$

If we move the contour of integration of (A.10) to the real axis, we get the expression for the Chern-Simons partition function computed in 21] using the Hermitian matrix model. Notice that in [53], when restricted to the case $S^{3}$, the partition function is then given by a single complex integral over the line $\mathbb{R} \times \mathrm{e}^{-\frac{i \pi}{4}}$, which is exactly (A.10) for that choice of contour.

We can use (A.9) and (A.10) to establish the relation between the unitary and Hermitian matrix models. If we rotate the contour of (A.9) to the imaginary axis [that means, taking the hook $\left.W_{-\frac{\pi}{2},-\frac{\pi}{2}}(s)\right]$, we get the unitary matrix model with $q=\mathrm{e}^{-g_{s}}$, by using the identities previously derived

$$
\begin{aligned}
& \int_{-\infty}^{\infty} \prod_{j<k}(-4) \sin ^{2}\left(\frac{u_{j}-u_{k}}{2}\right) \mathrm{e}^{-\frac{1}{2 g_{s}} \sum_{j=1}^{N} u_{j}^{2}} \prod_{j}(-i) \frac{d u_{j}}{2 \pi} \\
= & \mathrm{e}^{\frac{i \pi N(N+2)}{2}} \int_{-\infty}^{\infty} \prod_{j<k}\left|e^{i u_{j}}-e^{i u_{k}}\right|^{2} \mathrm{e}^{-\frac{1}{2 g_{s}} \sum_{j=1}^{N} u_{j}^{2}} \prod_{j} \frac{d u_{j}}{2 \pi} \\
= & N ! \mathrm{e}^{-\frac{i \pi N^{2}}{4}}(k+N)^{-\frac{N}{2}} \mathrm{e}^{-\frac{g_{s} N^{2}(N-1)}{12}} \prod_{j=1}^{N}\left(2 \sin \left(\frac{\pi j}{k+N}\right)\right)^{N-j} .
\end{aligned}
$$

By noting that (A.10) is precisely (A.1), then

$$
\begin{aligned}
Z & =\int_{0}^{2 \pi} \prod_{j} \frac{d u_{j}}{2 \pi} \Theta\left(q \mid u_{j}\right) \prod_{j<k}\left|\mathrm{e}^{i u_{j}}-\mathrm{e}^{i u_{k}}\right|^{2}=\left(\frac{2 \pi}{g_{s}}\right)^{\frac{N}{2}} \mathrm{e}^{-i \pi N(N+1)} \mathrm{e}^{-\frac{g_{s} N^{2}(N-1)}{6}} Z_{C S}^{U(N)}\left(S^{3}\right) \\
& =\mathrm{e}^{-i \pi N(N+1)} N ! \prod_{i=1}^{N-1}\left(1-q^{i}\right)^{N-i}
\end{aligned}
$$

This establishes the precise relation between the unitary and Hermitian Chern-Simons matrix models, derived directly from contour rotation and is our final result. It is worth mentioning that other integrals considered in [54] correspond to the Chern-Simons matrix model on $S^{3}$ but for other gauge groups. For example, Proposition 5.3.18 in [54] reads

Proposition 2: For $t \in \mathbb{Z}_{>0}$ we have

$$
\begin{aligned}
\widetilde{J}_{N, t}\left(\omega_{1}, \omega_{2}\right) \equiv & \frac{1}{\left(\sqrt{-\omega_{1} \omega_{2}}\right)^{N} N !} \int_{C} \prod_{j} \mathrm{e}^{\frac{t \pi i}{\omega_{1} \omega_{2}} x_{j}^{2}} d x_{j} \prod_{j<k} 4 \sin \left(\frac{\pi\left(x_{j}-x_{k}\right)}{\omega_{1}}\right) \sin \left(\frac{\pi\left(x_{j}-x_{k}\right)}{\omega_{2}}\right) \\
(\mathrm{A} .12) & \times \prod_{l<r} 4 \sin \left(\frac{\pi\left(x_{l}+x_{r}\right)}{\omega_{1}}\right) \sin \left(\frac{\pi\left(x_{l}+x_{r}\right)}{\omega_{2}}\right) \prod_{j=1}^{N}(-1) 4 \sin \left(\frac{\pi x_{j}}{\omega_{1}}\right) \sin \left(\frac{\pi x_{j}}{\omega_{2}}\right) \\
= & \frac{\mathrm{e}^{-\frac{i 3 \pi N}{4}}}{t^{\frac{N}{2}}} \mathrm{e}^{-\frac{\pi i N(N+1)(2 N+1)\left(\omega_{1}^{2}+\omega_{2}^{2}\right)}{6 t \omega_{1} \omega_{2}}} \prod_{j<k} 4 \sin \left(\frac{\pi(j+k)}{t}\right) \sin \left(\frac{\pi(j+k)}{t}\right) \prod_{j=1}^{N} 2 \sin \left(\frac{2 \pi j}{t}\right)
\end{aligned}
$$

where the contour of integration $C$ is a hook $W_{\phi_{1}, \phi_{2}}$ with $\phi_{1}, \phi_{2} \in \mathcal{A}_{+}$. This gives an evaluation for the partition function of the Chern-Simons matrix model for $S^{3}$ and the symplectic gauge group $S p(2 N)$. 


\section{REFERENCES}

[1] K. G. Wilson, "Confinement of quarks," Phys. Rev. D 10, 2445 (1974).

[2] L. Susskind, "Lattice Models Of Quark Confinement At High Temperature," Phys. Rev. D 20, 2610 (1979).

[3] M. Stone, "Lattice Formulation Of The Cp**(N-1) Nonlinear Sigma Models," Nucl. Phys. B 152, 97 (1979).

[4] P. Menotti and E. Onofri, "The Action Of SU(N) Lattice Gauge Theory In Terms Of The Heat Kernel On The Group Manifold," Nucl. Phys. B 190, 288 (1981).

[5] N. S. Manton, "An Alternative Action For Lattice Gauge Theories," Phys. Lett. B 96, 328 (1980).

[6] A. M. Polyakov, "Thermal Properties Of Gauge Fields And Quark Liberation," Phys. Lett. B 72, 477 (1978).

[7] M. E. Peskin, "Mandelstam 'T Hooft Duality In Abelian Lattice Models," Annals Phys. 113, 122 (1978).

[8] T. Banks, R. Myerson and J. B. Kogut, "Phase Transitions In Abelian Lattice Gauge Theories," Nucl. Phys. B 129, 493 (1977).

[9] E. Onofri, "SU(N) Lattice Gauge Theory With Villain's Action," Nuovo Cim. A 66, 293 (1981).

[10] D. J. Gross and E. Witten, "Possible third order phase transition in the large $N$ lattice gauge theory," Phys. Rev. D 21 (1980) 446-453.

[11] S. R. Wadia, "N = Infinity Phase Transition In A Class Of Exactly Soluble Model Lattice Gauge Theories," Phys. Lett. B 93, 403 (1980).

[12] O. Aharony, J. Marsano, S. Minwalla, K. Papadodimas and M. Van Raamsdonk, "The Hagedorn / deconfinement phase transition in weakly coupled large N gauge theories," Adv. Theor. Math. Phys. 8, 603 (2004) arXiv:hep-th/0310285

[13] I. R. Klebanov, J. M. Maldacena and N. Seiberg, "Unitary and complex matrix models as $1 D$ Type 0 strings," Commun. Math. Phys. 252 (2004) 275-323 arXiv:hep-th/0309168.

[14] A. Yu. Morozov, "Unitary integrals and related matrix models," Theor. Math. Phys. 162, 1 (2010) arXiv:0906.3518 [hep-thl]

[15] T. Okuda, "Derivation of Calabi-Yau crystals from Chern-Simons gauge theory," J. High Energy Phys. 0503 (2005) 047 arXiv:hep-th/0409270

[16] R. J. Szabo and M. Tierz, "Matrix models and stochastic growth in Donaldson-Thomas theory," arXiv:1005.5643[hep-th]]

[17] H. Ooguri, P. Sulkowski and M. Yamazaki, "Wall Crossing As Seen By Matrix Models," Commun. Math. Phys. 307 429, (2011) arXiv:1005.1293[hep-th]].

[18] J. Villain, "Theory of one-dimensional and two-dimensional magnets with an easy magnetization plane. 2. The Planar, classical, two-dimensional magnet," J. Phys. (France) 36 (1975) 581.

[19] J. V. Jose, L. P. Kadanoff, S. Kirkpatrick and D. R. Nelson, "Renormalization, vortices, and symmetry breaking perturbations on the two-dimensional planar model," Phys. Rev. B 16, 1217 (1977).

[20] E. Witten, "Quantum field theory and the Jones polynomial," Commun. Math. Phys. 121, 351 (1989).

[21] M. Tierz, "Soft matrix models and Chern-Simons partition functions," Mod. Phys. Lett. A 19, 1365 (2004) arXiv:hep-th/0212128

[22] R. J. Szabo and M. Tierz, "Chern-Simons matrix models, two-dimensional Yang-Mills theory and the Sutherland model," J. Phys. A 43, 265401 (2010) arXiv:1003.1228 [hep-th]]

[23] C. B. Lang, P. Salomonson and B. S. Skagerstam, "A Study Of Exactly Solvable Lattice Gauge Theories In Two Space-Time Dimensions," Phys. Lett. B 100, 29 (1981).

[24] C. B. Lang, P. Salomonson and B. S. Skagerstam, "Large N Lattice Gauge Theory Without Third Order Phase Transition," Nucl. Phys. B 190, 337 (1981).

[25] J. B. Kogut and L. Susskind, "Hamiltonian Formulation Of Wilson's Lattice Gauge Theories," Phys. Rev. D 11, 395 (1975).

[26] M. Aganagic, H. Ooguri, N. Saulina and C. Vafa, "Black holes, q-deformed 2D Yang-Mills, and nonperturbative topological strings," Nucl. Phys. B 715 (2005) 304-348 arXiv:hep-th/0411280]

[27] M. Blau and G. Thompson, "Derivation of the Verlinde formula from Chern-Simons theory and the G/G model," Nucl. Phys. B 408, 345 (1993) arXiv:hep-th/9305010].

[28] M. Blau and G. Thompson, "Chern-Simons theory on $S^{1}$-bundles: Abelianisation and $q$-deformed Yang-Mills theory," J. High Energy Phys. 0605 (2006) 003 arXiv:hep-th/0601068]

[29] I. Bars and F. Green, "Complete Integration Of U(N) Lattice Gauge Theory In A Large N Limit," Phys. Rev. D 20, 3311 (1979).

[30] L. P. Kadanoff, "Notes On Migdal's Recursion Formulas," Annals Phys. 100, 359 (1976).

[31] M. L. Mehta, Random Matrices (Academic Press, Boston, MA, 1991).

[32] W. Janke and H. Kleinert, "How Good Is The Villain Approximation?," Nucl. Phys. B 270, 135 (1986). 
[33] V. Periwal and D. Shevitz, "Unitary matrix models as exactly solvable string theories," Phys. Rev. Lett. 64, $1326(1990)$

[34] H. Ooguri and C. Vafa, "Worldsheet Derivation of a Large N Duality," Nucl. Phys. B 641, 3 (2002) arXiv:hep-th/0205297

[35] H. Neuberger, "Nonperturbative Contributions In Models With A Nonanalytic Behavior At Infinite N," Nucl. Phys. B 179, 253 (1981).

[36] J. Baik, R. Buckingham and J. DiFranco, "Asymptotics of Tracy-Widom distributions and the total integral of a Painlevé II function," Comm. Math. Phys., 280, 463 (2008) arXiv:0704.3636 [math.FA]]

[37] S. Cordes, G. W. Moore and S. Ramgoolam, "Lectures on 2D Yang-Mills theory, equivariant cohomology and topological field theories," Nucl. Phys. Proc. Suppl. 41 (1995) 184-244 arXiv:hep-th/9411210].

[38] A. A. Migdal, "Gauge transitions in gauge and spin lattice systems," Sov. Phys. JETP 42 (1975) 743.

[39] B. E. Rusakov, "Loop averages and partition functions in $U(N)$ gauge theory on two-dimensional manifolds," Mod. Phys. Lett. A 5 (1990) 693-703.

[40] C. Beasley and E. Witten, "Non-abelian localization for Chern-Simons theory," J. Diff. Geom. 70, 183 (2005) arXiv:hep-th/0503126

[41] C. Beasley, "Localization for Wilson Loops in Chern-Simons Theory," arXiv:0911.2687[hep-th]]

[42] X. Arsiwalla, R. Boels, M. Marino and A. Sinkovics, "Phase transitions in q-deformed 2d Yang-Mills theory and topological strings," Phys. Rev. D 73, 026005 (2006) arXiv:hep-th/0509002

[43] N. Caporaso, M. Cirafici, L. Griguolo, S. Pasquetti, D. Seminara and R. J. Szabo, "Topological strings and large $N$ phase transitions. I: Nonchiral expansion of $q$-deformed Yang-Mills theory," J. High Energy Phys. 0601 (2006) 035 arXiv:hep-th/0509041

[44] M. Marino, "Chern-Simons theory and topological strings," Rev. Mod. Phys. 77, 675 (2005) arXiv:hep-th/0406005

[45] J. Fuchs, Affine Lie Algebras and Quantum Groups: An Introduction, with Applications in Conformal Field Theory (Cambridge University Press, 1995).

[46] B. Kostant, "On MacDonald's $\eta$ - Function Formula, the Laplacian and Generalized Exponents," Adv. in Math., 20, 179-212 (1976).

[47] M. Aganagic, A. Neitzke and C. Vafa, "BPS microstates and the open topological string wave function," Adv. Theor. Math. Phys. 10 603-656 (2006) arXiv:hep-th/0504054

[48] E. Witten, "Gauge Theories, Vertex Models and Quantum Groups," Nucl. Phys. B 330, 285 (1990).

[49] S. de Haro and M. Tierz, "Brownian motion, Chern-Simons theory, and 2D Yang-Mills," Phys.Lett. B 601 (2004) 201-208 arXiv:hep-th/0406093

[50] S. Giombi, V. Pestun and R. Ricci, "Notes on supersymmetric Wilson loops on a two-sphere," JHEP 1007, 088 (2010) arXiv:0905.0665!hep-th]]

[51] M. Mariño, "Chern-Simons theory, matrix integrals, and perturbative three-manifold invariants," Commun. Math. Phys. 253 (2004) 25-49 arXiv:hep-th/0207096

[52] Y. Dolivet and M. Tierz, "Chern-Simons matrix models and Stieltjes-Wigert polynomials," J. Math. Phys. 48, 023507 (2007) arXiv:hep-th/0609167].

[53] R. Lawrence and L. Rozansky, "Witten-Reshetikhin-Turaev Invariants of Seifert Manifolds," Commun. Math. Phys. 205, 287-314 (1999).

[54] F. van de Bult, "Hyperbolic Hypergeometric Functions,", PhD Thesis, University of Amsterdam (2007).

Department of Physics

University of CALifornia SANTA Barbara

CALIFORNIA 93106, USA

E-mail address: mromo@physics.ucsb.edu

Departamento de Análisis Matemático, Facultad de Ciencias Matemáticas.

Universidad Complutense de Madrid.

Plaza de Ciencias 3, 28040, Madrid. Spain.

E-mail address: tierz@mat.ucm.es 
Grupo de Física Matemática

Complexo Interdisciplinar da Universidade de Lisboa

Avenida Professor Gama Pinto, 2, Pt-1649-003 Lisboa, Portugal.

E-mail address: tierz@cii.fc.ul.pt 